\title{
Full folding calculations for proton-nucleus elastic scattering at intermediate energies
}

\author{
R. Crespo, ${ }^{*}$ R. C. Johnson, and J. A. Tostevin \\ Department of Physics, University of Surrey, Guildford, Surrey GU2 5XH, United Kingdom
}

(Received 11 December 1989)

The full folding optical potential for the elastic scattering of protons from ${ }^{16} \mathrm{O}$ and ${ }^{40} \mathrm{Ca}$ at 200 $\mathrm{MeV}$ is calculated. The fully off-shell free nucleon-nucleon $t$ matrix, at a fixed energy, derived from the Paris potential and harmonic oscillator nuclear single-particle wave functions are used for both systems. The calculated observables show that the optimal factorization approximation provides a good description for both nuclei.

\section{INTRODUCTION}

In this paper we will examine the accuracy of the optimal factorization approximation, compared with the full folding approach, for calculations of the observables of proton elastic scattering from ${ }^{16} \mathrm{O}$ and ${ }^{40} \mathrm{Ca}$ at 200 $\mathrm{MeV}$ incident energy. Calculations in similar spirit have also been reported recently by Elster et al. ${ }^{1}$ for ${ }^{16} \mathrm{O}$, and by Arellano et al. ${ }^{2}$ for ${ }^{40} \mathrm{Ca}$. These calculations differ however, in detail, with each other with regard their physical input. For example, they use different underlying nucleon-nucleon $(N N)$ interactions, different prescriptions for the energy at which the $N N$ transition matrix is to be evaluated and different approximate treatments of the proton-target Coulomb interaction. Furthermore, the calculations appear to disagree on the basic question of the accuracy of the optimal factorization procedure.

Pauli blocking effects must be taken into account in order to understand quantitatively experimental data ${ }^{3}$ for proton elastic and inelastic scattering at intermediate energies. In finite nucleus calculations at intermediate energies, such effects are naturally taken into account within the Kerman-McManus-Thaler (KMT) multiple-scattering formalism. ${ }^{4}$ The inclusion of higher-order terms in the optical potential is, however, extremely complicated. It is essential, therefore, to investigate carefully the accuracy of the approximations currently made in the firstorder term, in particular the optimal factorization approach, ${ }^{5}$ which might lead to great simplifications in the evaluation of higher-order terms.

For these reasons, in this paper we have undertaken a consistent theoretical treatment of both the ${ }^{16} \mathrm{O}$ and ${ }^{40} \mathrm{Ca}$ systems, which uses the same underlying $N N$ and target structure models. Our calculations also differ from the published works in that they include a more accurate treatment of the proton-target Coulomb interaction. In view of the very significant differences between the predictions of the optimal factorization and full folding calculations, which were introduced by the addition of the Coulomb interaction in the work of Elster et al., it is important to clarify whether this phenomenon might result simply from the rather crude treatment of the Coulomb interaction used in that study.

\section{THE FIRST-ORDER OPTICAL POTENTIAL}

According to the KMT multiple-scattering formalism, the first-order optical potential for proton-nucleus scattering, in momentum space, is given by the expression

$$
\left\langle\mathbf{k}^{\prime}\left|\hat{U}^{(1)}\right| \mathbf{k}\right\rangle=\frac{A-1}{A} \sum_{\alpha_{i}, i=n, p}\left\langle\mathbf{k}^{\prime} \alpha_{i}\left|t_{0 i}(\varepsilon)\right| \mathbf{k} \alpha_{i}\right\rangle=\frac{A-1}{A} \sum_{\alpha_{i}, i=n, p} \int d \mathbf{p} \int d \mathbf{p}^{\prime}\left\langle\alpha_{i} \mid \mathbf{p}^{\prime}\right\rangle\left\langle\mathbf{k}^{\prime} \mathbf{p}^{\prime}\left|t_{0 i}(\varepsilon)\right| \mathbf{k} \mathbf{p}\right\rangle\left\langle\mathbf{p} \mid \alpha_{i}\right\rangle,
$$

where we have assumed that the target nucleus ground state is described by a single Slater determinant of occupied single-particle wave functions $\left\{\alpha_{i}\right\}$ and the sum, in Eq. (1), runs over all occupied states. Here $\mathbf{k}$ and $\mathbf{k}^{\prime}$ are projectile momenta in the nucleon-nucleus $(N A)$ center-of-mass frame, and $t_{0 i}(\varepsilon)$ is the antisymmetrized free nucleon-nucleon transition matrix evaluated at an appropriate relative energy $\varepsilon$ in the $N N$ center-of-mass frame. Additional effects, arising from the full antisymmetrization of the nucleon-nucleus optical potential, have been shown to be negligible. ${ }^{6}$

Using momentum conservation for the interacting nucleon pair, we write

$$
\left\langle\mathbf{k}^{\prime} \mathbf{p}^{\prime}\left|t_{0 i}(\varepsilon)\right| \mathbf{k} \mathbf{p}\right\rangle=\delta\left(\mathbf{k}^{\prime}+\mathbf{p}^{\prime}-\mathbf{k}-\mathbf{p}\right)\left\langle\frac{\mathbf{k}^{\prime}-\mathbf{p}^{\prime}}{2}\left|t_{0 i}(\varepsilon)\right| \frac{\mathbf{k}-\mathbf{p}}{2}\right\rangle,
$$

where

$$
\varepsilon=E-\frac{\hbar^{2}|\mathbf{k}+\mathbf{p}|^{2}}{4 m},
$$

$m$ is the nucleon mass and $E$ is the incident beam energy. If we now neglect target recoil effects and make the change of variable, $\mathbf{P}=\mathbf{p}-\mathbf{q} / 2$, we may write the full folding expression for the optical potential 


$$
\left\langle\mathbf{k}^{\prime}\left|\hat{U}^{(1)}\right| \mathbf{k}\right\rangle=\frac{A-1}{A} \sum_{\alpha_{i}, i=n, p} \int d \mathbf{P}\left\langle\alpha_{i} \mid \mathbf{P}-\frac{\mathbf{q}}{2}\right\rangle\left\langle\mathcal{K}^{\prime}\left|t_{0 i}(\varepsilon)\right| \mathcal{K}\right\rangle\left\langle\mathbf{P}+\frac{\mathbf{q}}{2} \mid \alpha_{i}\right\rangle,
$$

where $\mathbf{q}=\mathbf{k}^{\prime}-\mathbf{k}$ is the momentum transfer and $\mathcal{K}$ and $\mathcal{K}^{\prime}$, functions of $\mathbf{P}$, are the relative momenta of the two active nucleons, i.e.,

$$
\mathcal{K}=[\mathbf{k}-\mathbf{P}-\mathbf{q} / 2] / 2, \quad \mathcal{K}^{\prime}=\left[\mathbf{k}^{\prime}-\mathbf{P}+\mathbf{q} / 2\right] / 2 .
$$

If we neglect the momentum $p$ of the struck nucleon, in Eq. (3), and take for $k$ its on-shell value $k_{0}=\left(2 m E / \hbar^{2}\right)^{1 / 2}$, it follows that the required $N N$ transition matrix should be evaluated at an energy $\varepsilon=E / 2$, corresponding to free $N N$ scattering at half the beam energy in their center-of-mass frame. This is the approximation used in this paper.

\section{THE FINITE NUCLEUS MODEL}

In describing the target nucleus, we do not distinguish between protons and neutrons. We take the radial parts of the single-particle wave functions $\left\langle p \mid \alpha_{i}\right\rangle$ to be of harmonic oscillator (HO) form, denoted $R_{n_{\alpha} l_{\alpha}}(p)$, normalized such that

$$
\int p^{2} R_{n_{\alpha} l_{\alpha}}(p) R_{n_{\alpha}^{\prime} l_{\alpha}}(p) d p=\delta_{n_{\alpha} n_{\alpha}^{\prime}}
$$

The HO parameter $a$, see the Appendix for details, was obtained by fitting the calculated charge densities to the available electron-scattering data. ${ }^{7,8}$ The corresponding target matter densities were then deduced from this same length parameter.

\section{THE OPTIMAL FACTORIZATION APPROXIMATION}

For a closed-shell target nucleus, the result of the sum over the occupied states, in Eq. (4), is

$$
\left\langle\mathbf{k}^{\prime}\left|\hat{U}^{(1)}\right| \mathbf{k}\right\rangle=\frac{A-1}{A} \int d \mathbf{P}\left\langle\mathcal{K}^{\prime}\left|\bar{t}_{01}(\varepsilon)\right| \mathcal{K}\right\rangle \rho\left[\mathbf{P}-\frac{\mathbf{q}}{2}, \mathbf{P}+\frac{\mathbf{q}}{2}\right],
$$

where $\bar{t}_{01}(\varepsilon)$ is the spin and isospin averaged $N N$ transition matrix ${ }^{4}$

$$
\left\langle\mathcal{K}^{\prime}\left|\bar{t}_{01}(\varepsilon)\right| \mathcal{K}\right\rangle=\bar{A}\left(\varepsilon, \mathcal{K}^{\prime}, \mathcal{K}\right)+\sigma \cdot \hat{n} \bar{C}\left(\varepsilon, \mathcal{K}^{\prime}, \mathcal{K}\right),
$$

with $\sigma$ the Pauli spin matrix for the projectile, $\hat{n}=\mathcal{K} \times \mathcal{K}^{\prime} /\left|\mathcal{K} \times \mathcal{K}^{\prime}\right|$, and where

$$
\left.\left.\rho\left(\mathbf{P}-\frac{\mathbf{q}}{2}, \mathbf{P}+\frac{\mathbf{q}}{2}\right)=4 \sum_{n_{\alpha} l_{\alpha}} \frac{\left(2 l_{\alpha}+1\right)}{4 \pi} R_{n_{\alpha} l_{\alpha}}|| \mathbf{P}-\frac{\mathrm{q}}{2} \mid\right) R_{n_{\alpha} l_{\alpha}}|| \mathbf{P}+\frac{\mathbf{q}}{2} \mid\right) P_{l_{\alpha}}\left(\cos \theta_{P}\right) .
$$

In this expression $P_{l_{\alpha}}$ is the Legendre polynomial and $\theta_{P}$ is the angle between the vectors $\mathbf{P}+\mathbf{q} / 2$ and $\mathbf{P}-\mathbf{q} / 2$. The matrix elements of the two-body transition matrix $\left\langle\mathcal{K}^{\prime}\left|\bar{t}_{01}(\varepsilon)\right| \mathcal{K}\right\rangle$ are more conveniently expressed in terms of the momentum transfer $\mathbf{q}$ and the average of the relative momenta of the two nucleons $\mathcal{Q}=\left(\mathcal{K}^{\prime}+\mathcal{K}\right) / 2$, that is $\bar{t}_{01}(\varepsilon, \mathbf{q}, \mathbb{Q})$. Within this notation equation (7) can be rewritten as

$$
\left\langle\mathbf{k}^{\prime}\left|\hat{U}^{(1)}\right| \mathbf{k}\right\rangle=\frac{A-1}{A} \int d \mathbf{P} \bar{t}_{01}[\varepsilon, \mathbf{q}, \mathbf{Q}(P)] \rho\left[\mathbf{P}-\frac{\mathbf{q}}{2}, \mathbf{P}+\frac{\mathbf{q}}{2}\right),
$$

where the dependence of $\boldsymbol{Q}$ on $P$ has been made explicit.

The density matrix $\rho(\mathbf{P}-\mathbf{q} / 2, \mathbf{P}+\mathbf{q} / 2)$ is strongly peaked at $P=0$. Thus, assuming that the transition matrix $\bar{t}_{01}$ is a slowly varying function of $Q$, it has been suggested $^{5}$ that these matrix elements of the transition matrix should be fixed at $\bar{t}_{01}[\varepsilon, \mathrm{q}, Q(P=0)]$. This leads to the so-called optimal factorization form for the first-order optical potential

$$
\left\langle\mathbf{k}^{\prime}\left|\hat{U}^{(1)}\right| \mathbf{k}\right\rangle=U_{o f}^{c}\left(\mathbf{k}^{\prime}, \mathbf{k}\right)+\boldsymbol{\sigma} \cdot \hat{\mathbf{n}} U_{o f}^{l s}\left(\mathbf{k}^{\prime}, \mathbf{k}\right) .
$$

The central and spin-orbit components of the interaction are given by

$$
U_{o f}^{c}\left(\mathbf{k}^{\prime}, \mathbf{k}\right)=\frac{A-1}{A} \bar{A}(\varepsilon, \mathbf{q}, \mathbf{Q} / 2)[A \rho(q)],
$$

$$
U_{o f}^{l s}\left(\mathbf{k}^{\prime}, \mathbf{k}\right)=\frac{A-1}{A} \bar{C}(\varepsilon, \mathbf{q}, \mathbf{Q} / 2)[A \rho(q)]
$$

where $\mathbf{Q}=\left(\mathbf{k}+\mathbf{k}^{\prime}\right) / 2$ is the mean of the incoming and outgoing projectile momenta and $\hat{\mathbf{n}}$ the unit normal to the scattering plane in the $N A$ center-of-mass frame.

The nuclear matter density $\rho(q)$ is normalized such that $\rho(0)=1$. Explicitly, within the HO model,

$$
\rho(q)=\left[1-\frac{1}{8}(q a)^{2}\right] \exp \left(-q^{2} a^{2} / 4\right),
$$

and

$$
\rho(q)=\left[1-\frac{1}{4}(q a)^{2}+\frac{1}{80}(q a)^{4}\right] \exp \left(-q^{2} a^{2} / 4\right),
$$

for the ${ }^{16} \mathrm{O}$ and ${ }^{40} \mathrm{Ca}$ systems, respectively. 


\section{THE FULL FOLDING OPTICAL POTENTIAL}

In order to clarify the validity of the optimal factorization approximation described above, we will compare its predictions with those of the full folding calculations, obtained using the general method proposed by Redish and Stricker-Bauer. ${ }^{9}$ Defining $\phi$ as the angle between $q$ and $\mathcal{Q}$, the required matrix elements of $\bar{t}_{01}(\varepsilon)$ can be written

$$
\begin{aligned}
\bar{t}_{01}[\varepsilon, \mathrm{q}, \mathcal{Q}(P)] & =\bar{t}_{01}(\varepsilon, q, \mathcal{Q}, \phi) \\
& =\bar{A}(\varepsilon, q, \mathcal{Q}, \phi)+\boldsymbol{\sigma} \cdot \mathbf{N} \bar{C}^{\prime}(\varepsilon, q, \mathcal{Q}, \phi),
\end{aligned}
$$

where $\mathbf{N}=(\boldsymbol{Q} \times \mathbf{q}) / q \mathcal{Q}$. Thus, $\overline{\boldsymbol{C}}^{\prime}$ is related to the conventional spin/isospin averaged spin-orbit Wolfenstein amplitude $\bar{C}$, Eq. (8), according to $\bar{C}^{\prime}=\bar{C} / \sin \phi$. Evaluation of the off-shell components of $\bar{A}$ and $\bar{C}^{\prime}$ using realistic $N N$ interactions ${ }^{1,9}$ shows that for $N N$ relative momenta less than $3 \mathrm{fm}^{-1}$ and $50 \mathrm{MeV} \leq \varepsilon \leq 200 \mathrm{MeV}$, they are essentially independent of the variables $\varepsilon$ and $\phi$. Therefore, in order to simplify the integration over the angular variables of $\mathbf{P}$, it is convenient to perform a change of integration variable (to $\boldsymbol{Q}$ )

$$
\begin{aligned}
\left\langle\mathbf{k}^{\prime}\left|\hat{U}^{(1)}\right| \mathbf{k}\right\rangle=8 \frac{(A-1)}{A} \int & d \mathcal{Q} \bar{t}_{01}(\varepsilon, \mathbf{q}, \boldsymbol{Q}) \\
& \times \rho\left(\mathbf{k}-2 \boldsymbol{Q}, \mathbf{k}^{\prime}-2 \boldsymbol{Q}\right)
\end{aligned}
$$

and to then fix $\phi$ in the scattering amplitudes $\bar{A}$ and $\bar{C}^{\prime}$ to its on-shell value $\phi_{0}=\pi / 2$.

The density $\rho\left(\mathbf{k}-2 \mathbb{Q}, \mathbf{k}^{\prime}-2 \mathbb{Q}\right)$ depends only on $\mu$, the cosine of the angle between $\mathbb{Q}$ and $\mathbf{k}+\mathbf{k}^{\prime}$. In the evaluation of the integral over $Q$ we thus choose our $z$ axis along the direction $\mathbf{k}+\mathbf{k}^{\prime}$. The full folding integral can then be reduced to the standard form

$$
\left\langle\mathbf{k}^{\prime}\left|\hat{U}^{(1)}\right| \mathbf{k}\right\rangle=U_{f f}^{c}\left(\mathbf{k}^{\prime}, \mathbf{k}\right)+\boldsymbol{\sigma} \cdot \hat{\mathbf{n}} U_{f f}^{l s}\left(\mathbf{k}^{\prime}, \mathbf{k}\right) \text {. }
$$

The central component of the interaction is

$$
\begin{aligned}
& U_{f f}^{c}\left(\mathbf{k}^{\prime}, \mathbf{k}\right)=8 \frac{(A-1)}{A} \int \mathcal{Q}^{2} d \mathcal{Q} \bar{A}\left(\varepsilon, q, \mathcal{Q}, \phi_{0}\right) \\
& \times \sum_{n_{\alpha} l_{\alpha}} \frac{\left(2 l_{\alpha}+1\right)}{\pi} \mathcal{J}_{n_{\alpha} l_{\alpha}}^{c},
\end{aligned}
$$

with

$$
\begin{array}{rl}
\mathcal{J}_{n_{\alpha} l_{\alpha}}^{c}=2 \pi \int_{-1}^{1} & d \mu R_{n_{\alpha} l_{\alpha}}(|\mathbf{k}-2 Q|) R_{n_{\alpha} l_{\alpha}}\left(\left|\mathbf{k}^{\prime}-2 \boldsymbol{Q}\right|\right) \\
& \times P_{l_{\alpha}}\left(\cos \theta_{Q}\right)
\end{array}
$$

where $\theta_{\mathscr{Q}}$ is the angle between the vectors $\mathbf{k}^{\prime}-2 \mathbb{Q}$ and $\mathbf{k}-2 \boldsymbol{Q}$.

The spin-orbit component, after performing the azimuthal integration, ${ }^{1}$ is similarly given by the relation

$$
\begin{aligned}
U_{f f}^{l s}\left(\mathbf{k}^{\prime}, \mathbf{k}\right)= & 8 \frac{(A-1)}{A} \frac{\left|\mathbf{k} \times \mathbf{k}^{\prime}\right|}{Q q} \\
\times \int \mathcal{Q}^{2} d Q \bar{C}^{\prime}\left(\varepsilon, q, Q, \phi_{0}\right) & \\
& \times \sum_{n_{\alpha} l_{\alpha}} \frac{\left(2 l_{\alpha}+1\right)}{\pi} \mathcal{J}_{n_{\alpha} l_{\alpha}}^{l^{s}},
\end{aligned}
$$

where

$$
\begin{aligned}
\mathcal{J}_{n_{\alpha} l_{\alpha}}^{l s}=2 \pi \int_{-1}^{1} & \mu d \mu R_{n_{\alpha} l_{\alpha}}(|\mathbf{k}-2 Q|) R_{n_{\alpha} l_{\alpha}}\left(\left|\mathbf{k}^{\prime}-2 Q\right|\right) \\
& \times P_{l_{\alpha}}\left(\cos \theta_{Q}\right)
\end{aligned}
$$

Explicit formulas for the $g^{l s}$ and $g^{c}$ from the different occupied single-particle states, are presented in an appendix. Equivalent expressions for the optical potential in the case of ${ }^{16} \mathrm{O}$ have been presented by Elster et al. ${ }^{1}$

\section{THE ELASTIC-SCATTERING OBSERVABLES}

The optimal factorization, Eqs. (12) and (13), and the full folding expressions, Eqs. (19) and (21), for the firstorder optical potential were calculated in the case of proton elastic scattering from ${ }^{16} \mathrm{O}$ and ${ }^{40} \mathrm{Ca}$ at $200 \mathrm{MeV}$, using the off-shell $N N$ transition matrices calculated from the Paris potential. ${ }^{10,11}$ The target nucleus HO parameters used were $a=1.77 \mathrm{fm}$ and $a=1.95 \mathrm{fm}$, for ${ }^{16} \mathrm{O}$ and ${ }^{40} \mathrm{Ca}$, respectively. These parameters provide a reasonable description of the charge form factors for small momentum transfers, typically $q \leq 2 \mathrm{fm}^{-1}$.

In order to isolate those effects originating from the full treatment the off-shell character of the full folding optical potential, we first neglect the proton-target Coulomb interaction in the calculation of the observables. In Figs. 1 and 2 we demonstrate that the optimal factorization calculations of the proton elastic vector analyzing power $\left(A_{y}\right)$ angular distributions (dashed curves) are a good approximation to the full folding results (solid curves) for both the ${ }^{16} \mathrm{O}$ and ${ }^{40} \mathrm{Ca}$ systems. The differential cross-section angular distributions are not shown since the cross section shows very little sensi-

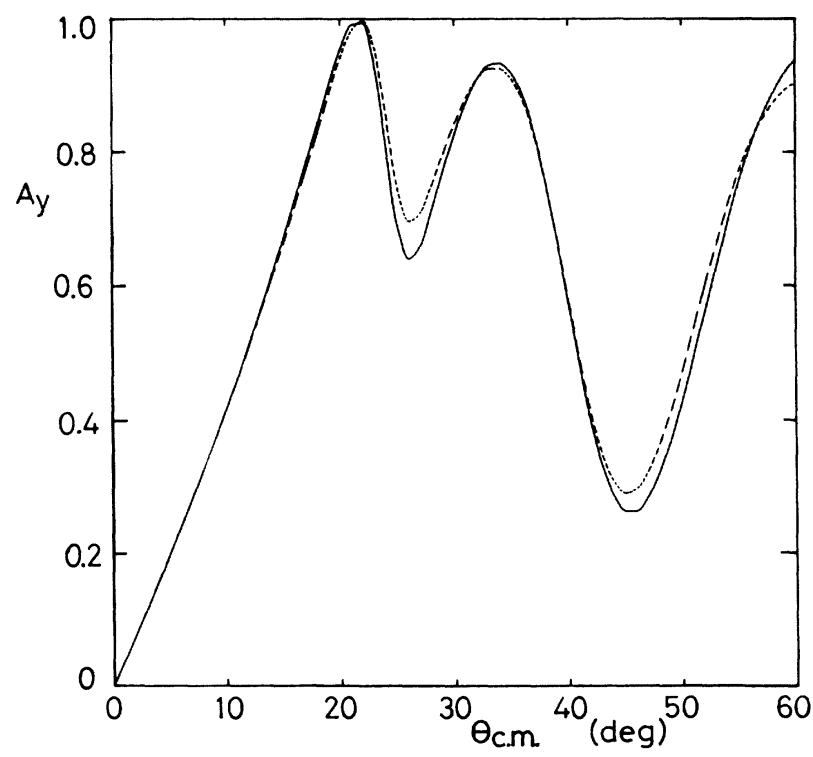

FIG. 1. Calculated vector analyzing powers $A_{y}$ for $p^{-16} \mathrm{O}$ elastic scattering at $200 \mathrm{MeV}$ in the absence of the Coulomb interaction. The dashed and solid curves were obtained using the optimal factorization and full folding optical potentials, respectively. 


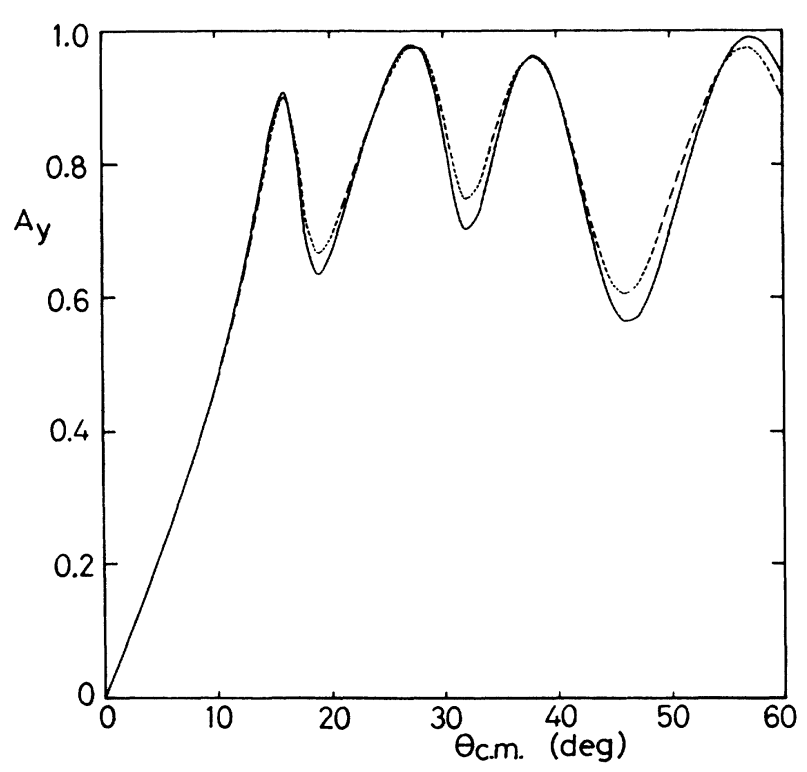

FIG. 2. Calculated vector analyzing powers $A_{y}$ for $p-{ }^{40} \mathrm{Ca}$ elastic scattering at $200 \mathrm{MeV}$ in the absence of the Coulomb interaction. The dashed and solid curves were obtained using the optimal factorization and full folding optical potentials, respectively.

tivity to the different approaches. Calculations were carried out, in momentum space, using the program LPOTPS. ${ }^{12}$ In the case of ${ }^{16} \mathrm{O}$, similar quantitative results were obtained using the $N N$ transition matrices derived from the Bonn potential. ${ }^{1}$ The small differences between our calculations show in the interference minima in $A_{y}$ and reflect a residual sensitivity to differences in the underlying $N N$ interactions at high momentum transfer and

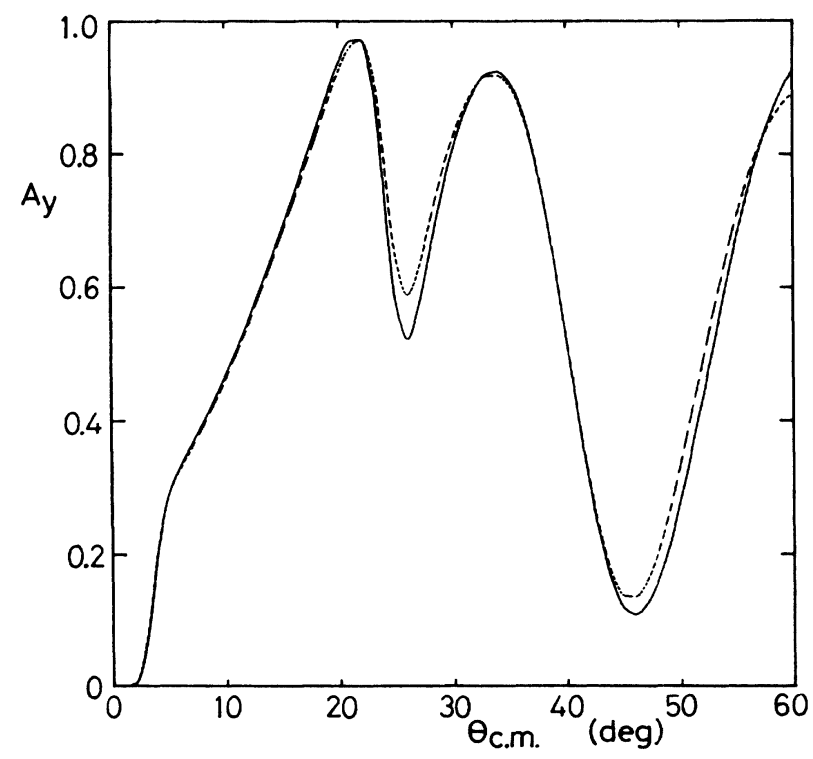

FIG. 3. As for Fig. 1, except that the Coulomb interaction is included using the subtracted momentum space method.

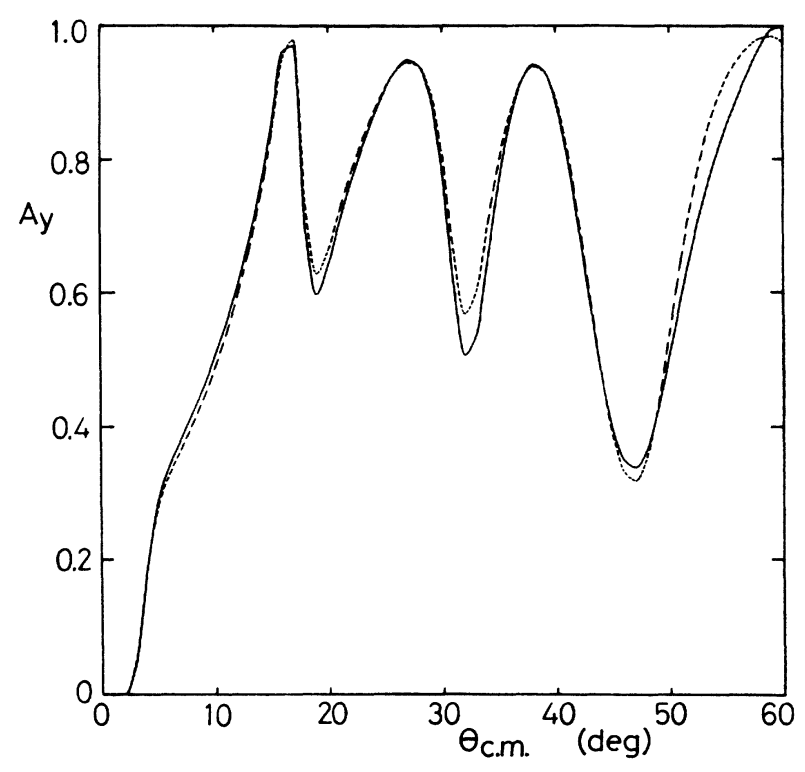

FIG. 4. As for Fig. 2, except that the Coulomb interaction is included using the subtracted momentum space method.

the slightly different values used for the single-particle HO range parameter $a$.

The Coulomb potential was included using the subtracted momentum space method ${ }^{13}$ which has been shown to produce accurate calculations of scattering observables. The Coulomb interaction was calculated from the calculated target charge densities. The cutoff radius for the Coulomb interaction, $R_{\text {cut }}$, used in our calculations was taken to be $R_{\text {cut }}=10 \mathrm{fm}$. This provided converged calculations of the proton analyzing power $A_{y}$ up to center-of-mass scattering angles of $70^{\circ}$.

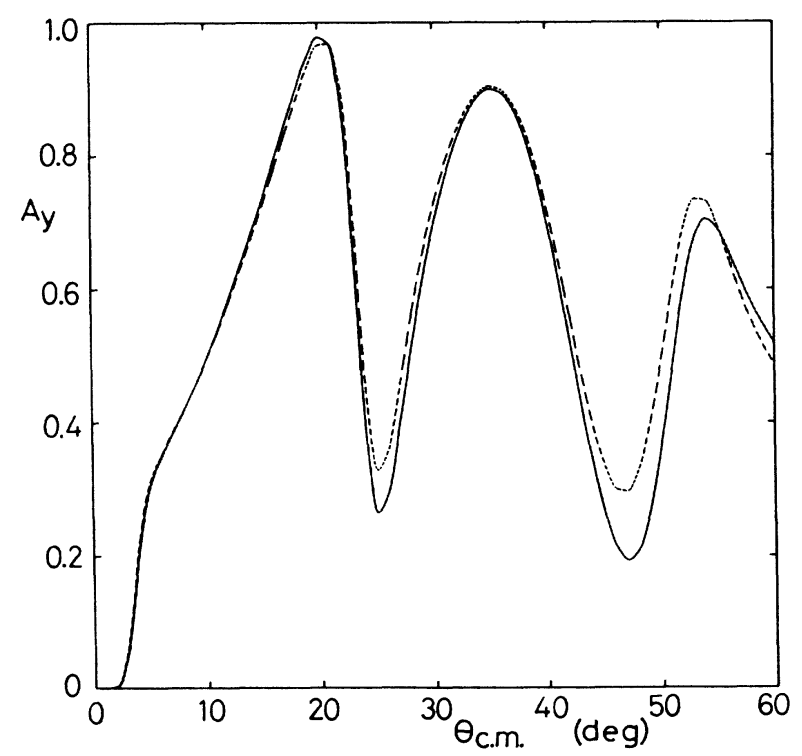

FIG. 5. As for Fig. 1 except that the Coulomb interaction is included using the technique adopted in Ref. 1. 
As is shown in Figs. 3 and 4, the inclusion of the Coulomb interaction does not enhance the differences between the full folding (solid curves) and optimal factorization (dashed curves) calculations. We find however, that if the Coulomb interaction is included only crudely, significant changes can be introduced between the two calculations. We demonstrate this effect for the ${ }^{16} \mathrm{O}$ system in Fig. 5, in which the Coulomb interaction has been treated adopting the method used by Elster et al. This was referred to as Method I in Ref. 13. It consists of approximating the partial wave transition amplitudes for scattering in the presence of the Coulomb interaction by purely nuclear amplitudes, the Coulomb interaction being introduced only through the Coulomb phase factor in the partial wave expansion of the scattering amplitude. We note that, not only does this technique for treating the Coulomb interaction magnify the differences between the optimal factorization (dashed curves) the full folding results (solid curves), it also introduces a deep first interference minimum in the calculated $A_{y}$ which does not arise with the more accurate Coulomb treatment. It is precisely in these interference minima that accuracy is required in order to study details of the calculated optical potentials and to investigate the need for the inclusion of higher-order terms, by comparison with the data.

In the work of Arellano et al. ${ }^{2}$ for the $p-{ }^{40} \mathrm{Ca}$ system at $200 \mathrm{MeV}$, it was concluded that the optimal factorization is not a good approximation to the full folding calculations. The difference between those calculations and those presented here, other than the use of different methods for the treatment of the Coulomb interaction, is the consideration, in Ref. 2, of the momentum of the struck nucleon.

\section{CONCLUSIONS}

In this paper we present calculations of elasticscattering observables for proton scattering from ${ }^{16} \mathrm{O}$ and ${ }^{40} \mathrm{Ca}$ at $200 \mathrm{MeV}$ using the first-order KMT optical potential. Calculations using the optimal factorization and full folding procedures have been carried out for both systems using consistent theoretical inputs for both systems together with an accurate treatment of the protontarget Coulomb interaction. We find that the optimal factorization procedure provides a very good approximation to the full folding results for both the ${ }^{16} \mathrm{O}$ and ${ }^{40} \mathrm{Ca}$ systems studied.

\section{ACKNOWLEDGMENTS}

The financial support of the Instituo Nacional de Investigação Científica and the British Council (for R.C.) and the Science and Engineering Research Council (U.K.) (SERC), through research Grant Nos. GR/E /6546.3, GR/F/4105.1, and GR/F/1086.6 (for R.C.J. and J.A.T.) is gratefully acknowledged. The authors would like to thank Professor E. F. Redish for his help in providing computer programs for the evaluation of the off-shell nucleon-nucleon transition matrix. We would also like to thank Dr. Ch. Elster, for her help in comparing calculations in the case of ${ }^{16} \mathrm{O}$, and Dr. E. D. (Tim) Cooper for many helpful discussions.

\section{APPENDIX}

In this Appendix we present explicit formulas for the harmonic-oscillator wave functions and the derived central and spin-orbit interaction integrals $\mathcal{I}_{n_{\alpha} l_{\alpha}}^{c}$ and $\mathcal{I}_{n_{\alpha} l_{\alpha}}^{l s}$ of Eqs. (20) and (22). Explicitly, the $R_{n_{\alpha} l_{\alpha}}(p)$ are

$$
\begin{aligned}
& R_{10}(p)=\widetilde{N}_{10} \exp \left(-p^{2} a^{2} / 2\right) ; \quad \tilde{N}_{10}^{2}=\frac{4 a^{3}}{\sqrt{\pi}}, \\
& R_{11}(p)=\widetilde{N}_{11} p \exp \left(-p^{2} a^{2} / 2\right) ; \quad \tilde{N}_{11}^{2}=\frac{8 a^{5}}{3 \sqrt{\pi}}, \\
& R_{12}(p)=\tilde{N}_{12} p^{2} \exp \left(-p^{2} a^{2} / 2\right) ; \quad \tilde{N}_{12}^{2}=\frac{16 a^{7}}{15 \sqrt{\pi}},
\end{aligned}
$$

and

$R_{20}(p)=\widetilde{N}_{20}\left(a^{2} p^{2}-\frac{3}{2}\right) \exp \left(-p^{2} a^{2} / 2\right) ; \quad \widetilde{N}_{20}^{2}=\frac{8 a^{3}}{3 \sqrt{\pi}}$.

For the $\mathcal{J}_{n_{\alpha} l_{\alpha}}^{c}$ and $\mathcal{J}_{n_{\alpha} l_{\alpha}}^{l s}$ we obtain, in the $1 S$ and $1 P$ states $\mathcal{I}_{10}^{c}=\tilde{N}_{10}^{2} G\left(k, k^{\prime}, x\right) j_{0}(z)$, $\mathcal{I}_{11}^{c}=\tilde{N}_{11}^{2} G\left(k, k^{\prime}, x\right)\left[\left(\mathbf{k} \cdot \mathbf{k}^{\prime}+x^{2}\right) j_{0}(z)+x\left|\mathbf{k}+\mathbf{k}^{\prime}\right| i j_{1}(z)\right]$,

where $j_{0}(z)$ and $j_{1}(z)$ are spherical Bessel functions, $x=2 Q, z=2 i \gamma x\left|\mathbf{k}+\mathbf{k}^{\prime}\right|, \gamma=a^{2} / 2$ and

$$
G\left(k, k^{\prime}, x\right)=4 \pi \exp \left[-\gamma\left(k^{2}+k^{\prime 2}+2 x^{2}\right)\right] .
$$

The $1 D$ and $2 S$ contributions are best evaluated together, in which case cancellation of terms simplifies the calculation. We find

$$
\begin{aligned}
5 \mathcal{J}_{12}^{c}+\mathscr{J}_{20}^{c}=G\left(k, k^{\prime}, x\right)\{ & \widetilde{N}_{20}^{2}\left[\frac{9}{4}-3 \gamma\left(k^{2}+k^{\prime 2}+2 x^{2}\right)\right] j_{0}(z)-\tilde{N}_{20}^{2} 6 \gamma x\left|\mathbf{k}+\mathbf{k}^{\prime}\right| i j_{1}(z) \\
& \left.+\widetilde{N}_{12}^{2} \frac{15}{2}\left[\left(\mathbf{k} \cdot \mathbf{k}^{\prime}+x^{2}\right)^{2}+x^{2}\left|\mathbf{k}+\mathbf{k}^{\prime}\right|^{2}\right] j_{0}(z)+\widetilde{N}_{12}^{2} \frac{15}{2} x\left|\mathbf{k}+\mathbf{k}^{\prime}\right|\left[2\left(\mathbf{k} \cdot \mathbf{k}^{\prime}+x^{2}\right)+\gamma^{-1}\right] i j_{1}(z)\right\}
\end{aligned}
$$

The corresponding spin-orbit integrals are

$$
\begin{aligned}
& \mathcal{J}_{10}^{l s}=-\widetilde{N}_{10}^{2} G\left(k, k^{\prime}, x\right) i j_{1}(z), \\
& \mathcal{J}_{11}^{l s}=-\widetilde{N}_{11}^{2} G\left(k, k^{\prime}, x\right)\left[\left(\mathbf{k} \cdot \mathbf{k}^{\prime}+x^{2}+\gamma^{-1}\right) i j_{1}(z)+x\left|\mathbf{k}+\mathbf{k}^{\prime}\right| j_{0}(z)\right],
\end{aligned}
$$

for the $1 S$ and $1 P$ states and 


$$
\begin{aligned}
5 \mathcal{J}_{12}^{l s}+\mathcal{I}_{20}^{l s}=-G\left(k, k^{\prime}, x\right)\{ & \widetilde{N}_{20}^{2}\left[-\frac{15}{4}-3 \gamma\left(k^{2}+k^{\prime 2}+2 x^{2}\right)\right] i j_{1}(z) \\
& +\widetilde{N}_{12}^{2} 15\left[\frac{1}{2}\left(\mathbf{k} \cdot \mathbf{k}^{\prime}+x^{2}\right)^{2}+\frac{1}{\gamma}\left(\mathbf{k} \cdot \mathbf{k}^{\prime}+x^{2}\right)+\frac{3}{4 \gamma^{2}}+\frac{x^{2}\left|\mathbf{k}+\mathbf{k}^{\prime}\right|^{2}}{2}\right] i j_{1}(z) \\
& \left.+\tilde{N}_{12}^{2} 15 x\left|\mathbf{k}+\mathbf{k}^{\prime}\right|\left[1+\frac{1}{2 \gamma}\right] j_{0}(z)-\widetilde{N}_{20}^{2} 6 \gamma j_{0}(z)\right\} .
\end{aligned}
$$

for the $1 D$ and $2 S$ contributions.

*On leave from Physics Department, Instituto Superior Técnico, Lisbon, Portugal.

${ }^{1}$ Ch. Elster, T. Cheon, E. F. Redish, and P. C. Tandy, Phys. Rev. C 41, 814 (1990).

${ }^{2}$ H. F. Arellano, F. A. Brieva, and W. G. Love, Phys. Rev. Lett. 63, 605 (1989).

${ }^{3}$ J. Hüfner and C. Mahaux, Ann. Phys. (N.Y.) 73, 525 (1972); J. P. Jeukenne, A. Lejeunne, and C. Mahaux, Phys. Rev. C 16, 80 (1977); L. Rikus and H. von Geramb, Nucl. Phys. A426, 486 (1984); C. Mahaux, ibid. A396, 9c (1983).

${ }^{4}$ A. K. Kerman, H. McManus, and R. M. Thaler, Ann. Phys. (N.Y.) 8, 551 (1959).

${ }^{5}$ A. Picklesimer, P. C. Tandy, R. M. Thaler, and D. H. Wolfe, Phys. Rev. C 30, 1861 (1984).

${ }^{6}$ L. Ray, Phys. Rev. C 39, 1170 (1989).

${ }^{7}$ T. W. Donnelly and G. E. Walker, Phys. Rev. Lett. 22, 1121 (1969).
${ }^{8}$ B. B. P. Sinha, G. A. Peterson, R. R. Whitney, I. Sick, and J. S. McCarthy, Phys. Rev. C 7, 1930 (1973).

${ }^{9}$ E. F. Redish and K. Stricker-Bauer, Phys. Rev. C 35, 1183 (1987).

${ }^{10}$ M. Lacombe, B. Loiseau, J. M. Richard, R. Vinh Mau, J. Côté, P. Pires, and R. de Tourreil, Phys. Rev. C 21, 861 (1980).

${ }^{11}$ E. F. Redish and K. Stricker-Bauer, Phys. Rev. C 36, 513 (1987).

${ }^{12}$ Program LPOTPS is an extensively revised and extended version of the program LPOTP; M. J. Paez, M. E. Sagen, and R. H. Landau, Comput. Phys. Commun. 52, 141 (1988).

${ }^{13}$ R. Crespo and J. A. Tostevin, Treatment of the Coulomb interaction in momentum space calculations of proton elastic scattering, University of Surrey report, 1989 (to appear in Phys. Rev. C). 